\title{
Exoplanet imaging: from ELT's to interferometers and hypertelescopes
}

\author{
Antoine Labeyrie \\ College de France \& Observatoire de Calern, 06460 Caussols, France \\ email: antoine.labeyrie@obs-azur.fr
}

\begin{abstract}
ELT's, interferometers and their multi-aperture direct-imaging form called hypertelescopes are expected to provide images of exo-Earths, and even resolved images in the latter case. Both terrestrial and space versions are potentially usable, although high-performance adaptive optics will be needed on Earth. Current advances in the way of hypertelescopes on Earth and in space are briefly described.
\end{abstract}

Keywords. exoplanets, interferometer, hypertelescopes.

\section{Introduction}

Following the discovery of more than 160 exo-planets, with the indirect method of "wobble spectrography", obtaining exo-planet images is becoming a major challenge for astronomy. Three decades after the proposal of a coronagraphic camera for exo-planet imaging with the Hubble Space Telescope (Labeyrie (1974), Bonneau et al. (1975), NASA is planning TPF-C a Hubble-class space telescope dedicated for exo-planet coronagraphy, and many groups now explore various ways of achieving enough detection sensitivity for imaging Earth-like planets, also called exo-Earth's.

\section{Telescopes, interferometers and hypertelescopes for exo-planet imaging}

These three types of instruments, operated on Earth or in space, can potentially serve for exo-planet imaging. Telescopes can have a monolithic mirror, for which the size limit is considered to be of the order of 8 meters, or a mosaic mirror which can be much larger, up to a hundred meters according to the conclusions of ESO's study work for their OWL concept. Interferometers, using several telescopes or separately mounted mirrors, can reach much larger sizes, and therefore attain a higher resolution. The information content of the snapshot exposures can however be very limited if there are few apertures. In principle, however, it can be improved with multiple-exposures, according to the aperture synthesis and super-synthesis techniques. These have been rather successful at radio wavelengths, but not yet much utilized in the optical and near infra-red ranges. Hypertelescopes provide a way of bridging the gap between the telescope and interferometer realms, by using many sub-apertures forming an aperture which can be highly diluted while retaining a capability to produce snapshot images like conventional telescopes. Their imaging capability includes coronagraphic modes and therefore makes them suitable for obtaining multi-resel images of exo-Earths with arrays becoming as large as $100 \mathrm{~km}$. 


\section{Principle and properties of hypertelescopes}

These have been described elsewhere (Le Coroller et al., 2004). Here we only recall

\section{Types of hypertelescope architectures}

The principle of hypertelescopes, described elsewhere, can be materialized in several different practical architectures. These may be classified as using a primary array having either a planar shape, or a paraboloidal shape, or else a spherical shape.

\subsection{Planar hypertelescopes}

These utilize an array of telescopes, each equipped with a Coudé train, possibly fibered, to bring the captured light to a common focus. Coherencing is achieved either by moving the telescopes, or with delay lines such as those installed at the Keck, the VLTI, the CHARA, etc. The high cost of such moving telescopes or delay lines limits the number of apertures which can be used and the baseline span. In space, no such restriction applies since planar telescope arrays can be globally pointed, but the concave mirror arrays discussed below may then provide simplified optical solutions.

\subsection{Spherical hypertelescopes}

This concept, called Carlina, has a primary array of mirrors located on a common spherical surface. On Earth, natural rocky sites such as extinct volcano craters, canyons or sink holes can have suitable shapes to support many fixed mirrors, adjusted co-spherically. A corrector of spherical aberration, typically a two-mirror clam-shell arrangement such as the Mertz corrector which also corrects coma, provides a usable image near the focal surface, located half way to the curvature center.

\subsection{Pointable paraboloidal hypertelescopes}

The corrector of spherical aberration can be avoided if the primary locus of the mirror segments is paraboloidal rather than spherical. But the aberration-free field of view then becomes very narrow around the axial direction. A pointing mount is therefore necessary to attain a useful sky coverage. On Earth, conventional pointing mounts cannot be made much larger than $100 \mathrm{~m}$ however, and can certainly not reach the kilometric size which would be desirable for high resolution imaging. Figure 2 sketches a putative solution involving mirror segments supported by a net-like structure which is suspended and pointable by pulling cables. This version, called Galanthus, is of interest for the Dome $\mathrm{C}$ in Antarctica, where the winds are very low but with a significant ground turbulence justifying a $40 \mathrm{~m}$ altitude for the mirrors. The suspension can be from balloons, three at least, or from fixed structures such as canyon walls, skyscrapers, pylons, etc...

\section{Comparison of hypertelescope architectures}

For planar arrays, the cost of telescopes and movable optical elements in the Coudé trains obviously limits the aperture count to a few units. This severely restricts the imaging performance of such instruments, particularly in terms of the allowable object complexity. On Earth, the adoption of a spherical architecture is highly dependant on the availability of suitably shaped sites, also having desirable astronomical characteristics, accessibility, infra-structure, etc. Craters, either extinct volcanoes, meteorite impact craters or karstic depressions, can be exploited like the sink hole where the $330 \mathrm{~m}$ wide Arecibo radio telescope is nested. Instead of its high pylons, a balloon can serve to suspend the focal optics. Several stars can be observed simultaneously with as many focal 
correctors, carried independently. The number of mirrors can reach in the hundreds or thousands. Their anchoring to the bed-rock provides a more stable optical adjustment than with other architectures. Three micrometric screws carrying each mirror can be manually adjusted at months intervals if the bed rock is reasonably stable. The size of the focal corrector for spherical aberration increases as the third power of the focal ratio, reaching about $1 \%$ of the primary size at $\mathrm{F} / 2$. This limits in practice the design possibilities to focal ratios slower than F/1.5. For a $1.5 \mathrm{~km}$ effective aperture, achievable in principle at sites such as the Taburiente crater ( Canary Islands), $12 \mathrm{~m}$ mirrors are needed for the corrector, and these can be made in the form of a balloons reflective membrane. In space, effective apertures larger than $100 \mathrm{~km}$, and probably up to $100,0000 \mathrm{~km}$, appear feasible.

Paraboloidal architectures are limited in aperture size, on Earth, to $100 \mathrm{~m}$ with conventional pointing mounts and perhaps one kilometer with the net suspension structure. The mirror segments have shapes which differ according to their distance from the axis. As experienced with the segments of the Keck telescopes, this makes them more costly than uniform spherical segments. At a flat site such as Antarctica's Dome C, where wind velocities are often exceptionally low, balloons are of particular interest for suspending the net, which can be located above the ground layer, for improved seeing.

\section{Directly imaging exo-planets with these instruments}

Three decades after the early proposal of an exo-planetary coronagraph attached to the Hubble Space telescope NASA is now planning a dedicated coronagraphic and exoplanetary telescope named TPF-C. Current studies indicate that a $3 \mathrm{~m}$ aperture telescope, equipped with "extreme" forms of coronagraphic cleaning can provide exo-Earth images at visible wavelengths. But these images will not have enough resolution to show any details of these planets. Low-resolution spectra of the planets integrated light can be expected to provide clues on the presence of gas molecules indicative of life, such as O2,O3, $\mathrm{CO} 2, \mathrm{H} 2 \mathrm{O}$, but none of these molecules can be considered as a robust criterion: the photodissociation of $\mathrm{H} 2 \mathrm{O}$, possibly catalyzed by certain minerals, can in principle generate $\mathrm{O} 2$, O3, and CO2. Resolving planetary details is desirable to reach more robust criteria for the presence of life. Local, at the scale of regions, and seasonal variations of cloud cover and absorbtion bands analog to the Earths chlorophyll features, can provide more useful information. This requires large baselines, beyond $100 \mathrm{~km}$ at visible wavelengths, which themselves require space instruments.

\section{Resolving the details of exo-Earths with hypertelescopes in space: the Exo-Earth Imager}

Obtaining resolved images of exo-Earths with enough detail to attempt the detection of life forms requires, in addition to baselines longer than $100 \mathrm{~km}$, a deep rejection of light from the parent star. For fast observations, providing snap-shot images within $30 \mathrm{mn}, 100$ or more apertures are needed and their size should be $3 \mathrm{~m}$ at least. With hypertelescope imaging, a larger number of smaller apertures can provide a better result at equal collecting area. In terms of the hypertelescope architecture to be used in space, the paraboloidal and spherical types are especially relevant. The former requires global pointing whenever a new star is to be observed, a process which is necessarily long with a formation flight spanning $100 \mathrm{~km}$ or more. The latter can have a stationary formation flight of primary mirrors if these are arranged as a full, but diluted sphere. Only the focal optics needs to move in order to explore the focal sphere. However, this 


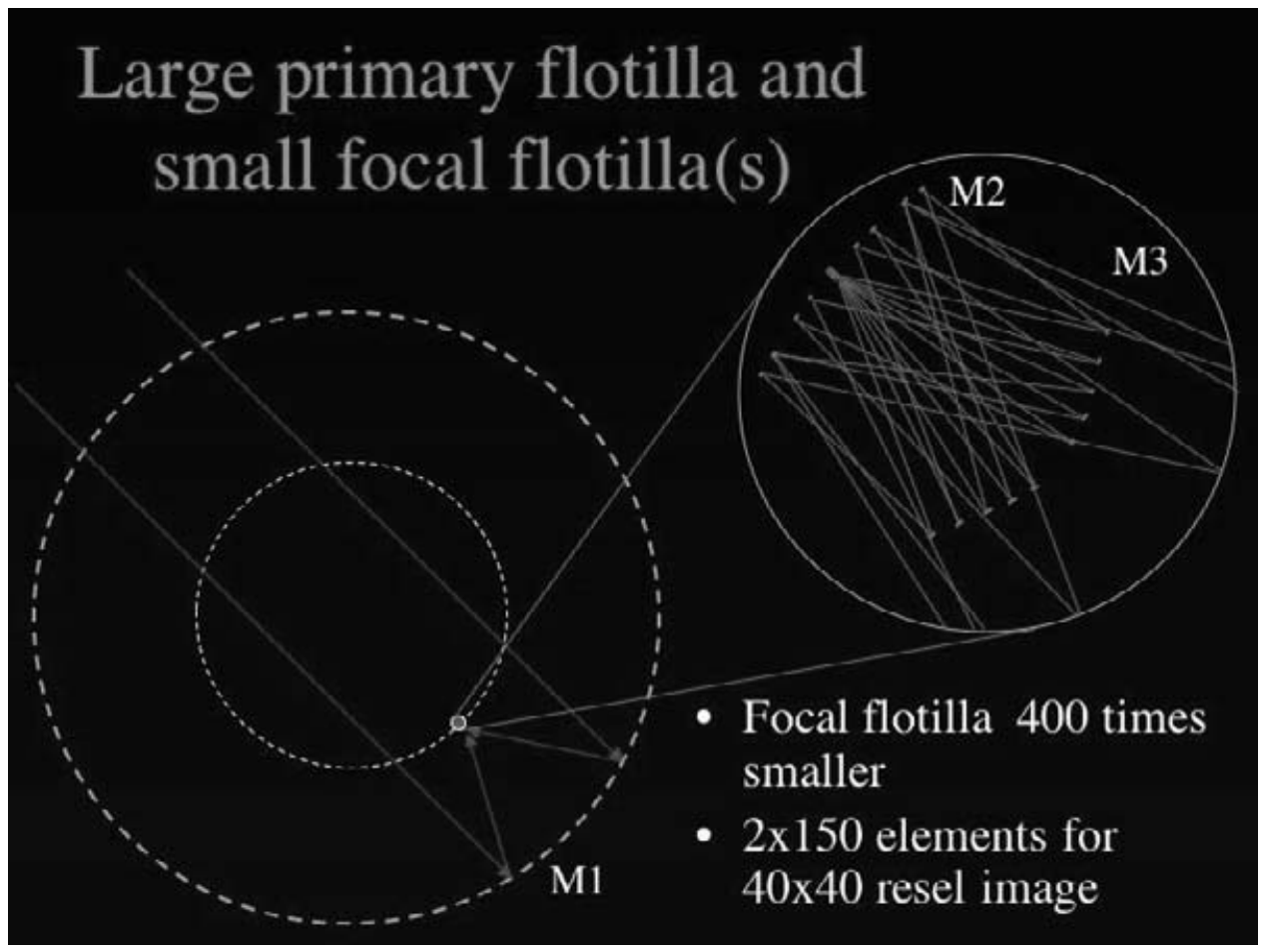

Figure 1. Concept of the Exo-Earth Imager (EEI), a large space version of spherical hypertelescope intended for imaging exo-Earths. It includes 10,000 mirrors of 3m, arranged as a spherical bubble with $400 \mathrm{~km}$ diameter. The focal sphere, half way to the center, is explored by one or more beam-combiner systems which include an aberration corrector in the form of a smaller formation flight, $1 \mathrm{~km}$ in size and containing $10 \mathrm{~cm}$ mirrors. The large diluted spherical mirror is static, and there can be several static focal flotillas as well, dedicated to the permanent observation of favorite exo-planets.

concept requires correcting optics which itself should be made in the form of a smaller formation flight, spanning a kilometer or more, although with mirror elements as small as $100 \mathrm{~mm}$. A spherical hypertelescope concept proposed along these lines, called ExoEarth Imager, is under preliminary study. A precursor instrument, called Luciola, is also studied for developing suitable techniques of formation flight control, while performing high-resolution snapshot imaging of stellar surfaces.

\section{Exo-Earth coronagraphy with ELTs, TPF-C and Exo-Earth Imager}

Techniques of extreme coronagraphy are needed to obtain images of exo-Earths at visible wavelengths, where their relative luminosity approaches a billionth. Some of the apodizer and coronagraph systems can in principle reach a complete nulling of the residual stellar light, assuming: a- perfect optics devoid of any surface bumpiness; and b- a nonresolved star. But neither condition is fulfilled in practice: a- mirrors, even corrected with high-order adaptive optics, have residual bumpiness diffracting significant levels of residual star light in the form of speckles contaminating the planet's image; b- stars are slightly resolved in telescopes larger than a few meters, at visible wavelengths.

Further cleaning with coherent or incoherent techniques is therefore needed for a deeper nulling of the star's residual speckles. Coherent techniques can use actuators located: 
a- before the focal plane: Trauger et al. ( this conference) have shown that a very accurate adjustment of the actuators can clean the coronagraph output image down to levels suitable for detecting Jupiters. It is unclear however whether the method is affected by the partial resolution of the star. b- after the focal plane, within a coronagraph, using destructive interference to null the residual stellar speckles. This is achievable in different ways using for example a multi-resel version of Bracewells nulling method, with a spatial filter in the form of a fiber bundle, or a Mach-Zehnder interferometer, or a dynamic hologram. These methods relax the needed actuator accuracy. The effect of the star's partial resolution remains to be assessed, and ways of reaching a usable spectral bandwidth have to be developed.

Incoherent cleaning is performed post-detection, typically by subtracting a reference image of a star having no planet. Unlike coherent cleaning, which can in principle achieve a complete null, it leaves in each speckle a residue level, the ultimate value of which corresponds to the photon-noise, amounting to the square root of the number of recorded photons. Incoherent cleaning should therefore be applied once the best possible coherent cleaning has been achieved.

These coronagraphic techniques are applicable to conventional telescopes and ELT's, to interferometers and to hypertelescopes. With the future very large arrays needed to image the details of exo-Earth's, such as the Exo-Earth Imager, coherent cleaning is likely affected by the fact that the planet is highly resolved. But the method can be applied before combining the beams, by using a separate coronagraph for each sub-aperture. On Earth, highly accurate adaptive optics is needed and it is difficult to predict how the planet detection sensitivity of ELTs will compare with that of space instruments.

\section{Progress of Carlina hypertelescopes}

Following the first fringes obtained on Vega by Le Coroller et al. (2004) with a partly completed Carlina prototype, J.Dejonghe has installed a Mertz corrector on the instruments gondola. It has been partially tested on stars. Also, the three ground mirrors have been re- positioned to provide baselines of $10.5 \mathrm{~m}, 9 \mathrm{~m}$ and $5 \mathrm{~m}$.

One of the dolines at Calern Observatory is considered for a larger Carlina II version. A contour map with $10 \mathrm{~cm}$ accuracy, generated by aerial photogrammetry, indicates that an effective aperture size of 40 to $60 \mathrm{~m}$ can be exploited at this site. Canyon sites, in the southern Alps and elsewhere, are also considered for larger effective apertures, reaching a few hundred meters. Some of them can be equipped with a cable stretched across, which can make the balloon unnecessary.

\section{Feasibility study of Galanthus, a paraboloidal hypertelescope for Dome C}

The Galanthus genus of flowers includes G. nivalis, known as the Snowdrop in English and Perce-neige in French. It does not exist at Dome $\mathrm{C}$, but may lends its name to a flower-like paraboloidal hypertelescope especially suited for this site, having a flat topography and very low wind Figure 2. Unlike the "grounded" Carlina mirrors, the suspended mirrors cannot be expected to keep a sufficiently stable geometry by passive means only. Each mirror is therefore carried by three actuators, under control from a real-time metrology device, such as a laser theodolite installed at the focal gondola. The complete pointable optical structure is expected to be located above the turbulent ground layer, said to be less than 40m thick. The flexural behavior of the net structure, particularly as a function of the pointing elevation, and its oscillations should now be modeled 


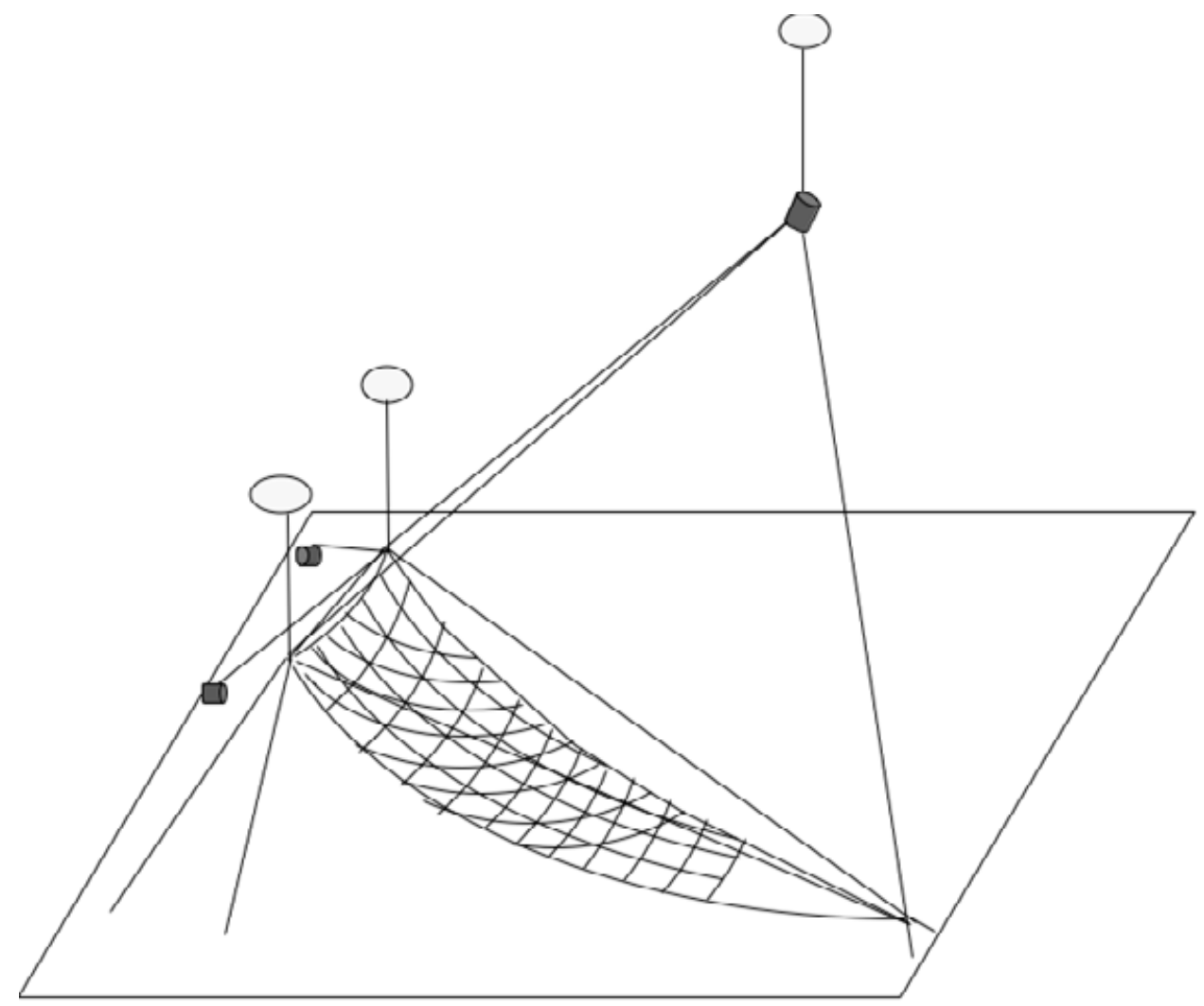

Figure 2. Scheme of Galanthus, a pointable paraboloidal hypertelescope for Dome C, Antarctica. A net-like structure stretched at the bottom of a tetrahedron, both made of high-modulus tensioned cable, is suspended 40m above the snow surface from three balloons. Small lightweight mirrors, figured as paraboloidal segments, are supported at each node of the net by three actuators for active geometry corrections. Tethers, actuated by computerized winches, control the pointing direction. The focal optics, including a pupil densifier, coronagraph, adaptive optics, camera, etc.. is located at the upper tip of the tetrahedron, although part of it can be located at ground level in a Coude laboratory.

with finite- elements code. This is needed to calculate the maximal aperture size, which may be of the order of a kilometer. In comparison with Carlina architectures, Galanthus has the drawback of strongly relying on active corrections, but advantages of not requiring a corrector of spherical aberration nor an exceptional concave site topography. Its focal ratio can also be shorter, $\mathrm{F} / 1$ instead of $\mathrm{F} / 1.7$ for Carlinas. A delicate issue is to manage the occasional landing of the structure, for storm protection and maintenance. There is some controversy on the statistics of storms at Dome C. Also, the emission of polluting soot particles by the local power station is a major concern for astronomical projects at the site.

\section{Design progress for a Luciola hypertelescope in space}

Luciola is a "bare bone", and much smaller, precursor version of an Exo-Earth Imager which can be flown in the near future to verify the concept and, if successful, provide direct resolved images of comparatively easy objects such as the nearby stars. The brighter exo-planets, such as the hot Jupiters associated to $51 \mathrm{Peg}$ and Tau Boo are also 


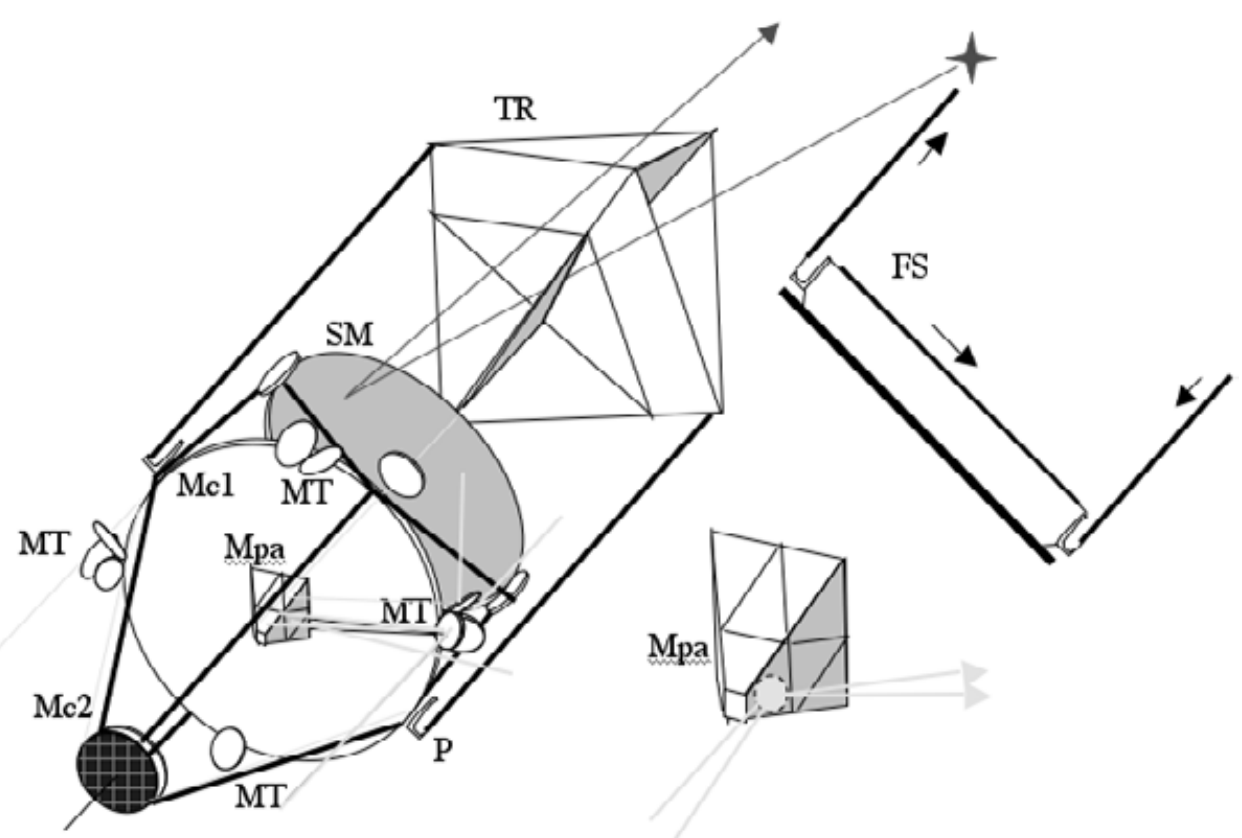

Figure 3. Concept of Luciola spaceships carrying elements of the giant diluted mirror. The low mass structure is translated and rotated by a small rigid solar sail, configured as a Ritchey-Chretien telescope (mirrors Mc1 and Mc2). It is pointed towards the Sun and focuses its image on the faceted pyramid mirror Mpa, mounted at the center of Mc1 and having 16 concave facets which relay pupil images on corresponding facets of the peripheral trihedral mirrors MT. Mc2 is controlled in tip-tilt by two actuators for switching the light output direction, by addressing different facets of Mpa. The tip-tilt actuators can remain nearly inactive during normal observing since the four central facets of Mpa feed those peripheral MT facets which reflect the beam in the anti-solar direction. The resulting torque tends to correct pointing errors passively. The actuators are needed to control all six force and torque components applied to the satellite. In the event of a complete loss of sun pointing, the sun image falls outside of Mpa and it becomes ineffective for attitude control. The tail reflector TR then becomes illuminated and applies a restoring torque, which tends to re-point the Cassegrain optics towards the Sun. Oscillations are damped by a damped flexural swivel FS connecting TR to the main structure. The stellar mirror SM is nearly flat and located in the shadow behind Mc1, also serving as a sunshield. It has one rotation axis, activated by a micro-motor. The second rotation needed to point a star is achieved by rotating the entire satellite about the Sun's direction, using the MT facets which provide a tangential output beam. SM has a central hole with a miniature EM-CCD camera serving for stellar pointing. The satellite has a low mass, typically a few hundred grams for a $20 \mathrm{~cm}$ SM mirror, allowing sufficient accelerations with solar radiation pressure.

potential targets. On Earth, Luciola is also a genus of nocturnal firefly beetles, seen as dancing swarms with spectacular flashing displays. Here, it alludes to the flashing swarm of free-flyers equipped with pulsed diode lasers. These serve to determine the swarm's geometry,using time of flight measurements.

The segments of the diluted primary mirror are carried by nano-satellites driven by small solar sails for economy, for a long-life, and for avoiding the exhaust plumes of conventional thrusters, which tend to contaminate the cold mirrors. The scale size considered for Luciola is $20 \mathrm{~cm}$ for the mirrors and a few hundred meters for the array aperture. Figure 3 shows the optical concept, the solar sail part of which is based on a Zemax ray-trace by David Vernet and Alain Blazit. 
Each satellite emits encoded light pulses, non directional, and detects pulses from other satellites, using simple non-directional detectors. From the detected signals, the internal geometry of the swarm can be calculated with millimeter accuracy. A laser theodolite installed on the focal satellite, or a white-light interferometer installed at the curvature center can also provide such metrologic information, but can be more costly and difficult to operate. In the latter case, the co-sphericity errors of the primary segments can be measured with nanometric accuracy.

The solar radiation pressure is very weak, slightly less than a microNewton on a $20 \mathrm{~cm}$ mirror, but the response is highly linear and predictable, with no stick-slip, hysteresis or other problems of earthly actuators. In the absence of wind and the other disturbances affecting Earth based interferometers, it is therefore expected that the sail system can maintain the position of the stellar mirror within Rayleigh's tolerance, and possibly better. If not, a conventional adaptive deformable mirror will be needed in the focal package to achieve proper phasing.

\section{Conclusions}

Connsiderable progress is under way towards forming images of exo-Earths, and then high-resolution images showing their detail, at the micro-arc-second scale, in multi-resel images. Established spectro-imaging techniques will provide spectra of each resel in such images, and this can help searching life through chlorophyll-like absorbtion bands and their seasonal variation. Current efforts in our group are directed towards building precursor versions of hypertelescopes on Earth and in space. Among these versions, the Carlina II and the Luciola are rather modest in size and cost, but expected to teach us how to build much larger hypertelescopes with effective aperture diameters reaching $1500 \mathrm{~m}$ on Earth and 100 to $100,000 \mathrm{~km}$ in space. These aperture sizes in space are expected to respectively resolve exo-Earths and neutron stars.

\section{References}

Labeyrie, A. 1974, proposal to NASA

Bonneau, D., Josse, M., \& Labeyrie, A. 1975, in: Nieuwenhuijzen \& de Jager (eds.), Image processing techniques in Astronomy, D. Reidel, (Holland), p. 403

Le Coroller, H., Dejonghe, J., Arpesella, C., Vernet, D., \& Labeyrie, A. 2005, Astron.Astrophys. vol. 426 , p. $721-28$ 